Suzanne de Lotbinière-Harwood

\title{
Extrême centre
}

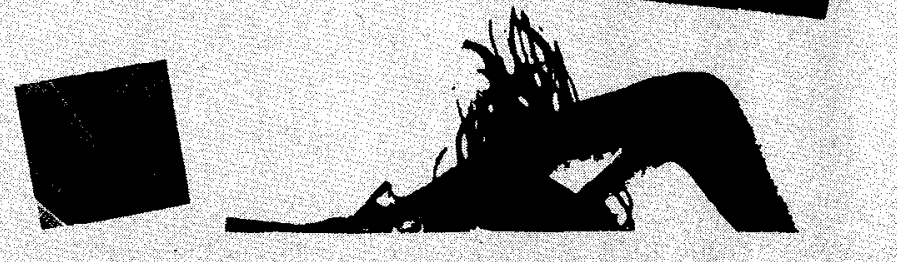




\section{à François Truffaut}

- what do you want out of me? she said

- undulating beneath ber i cried - lust!

- what do you want out of me, $i$ asked her.

- language, she said, language.

Patti SMITH ${ }^{1}$ 


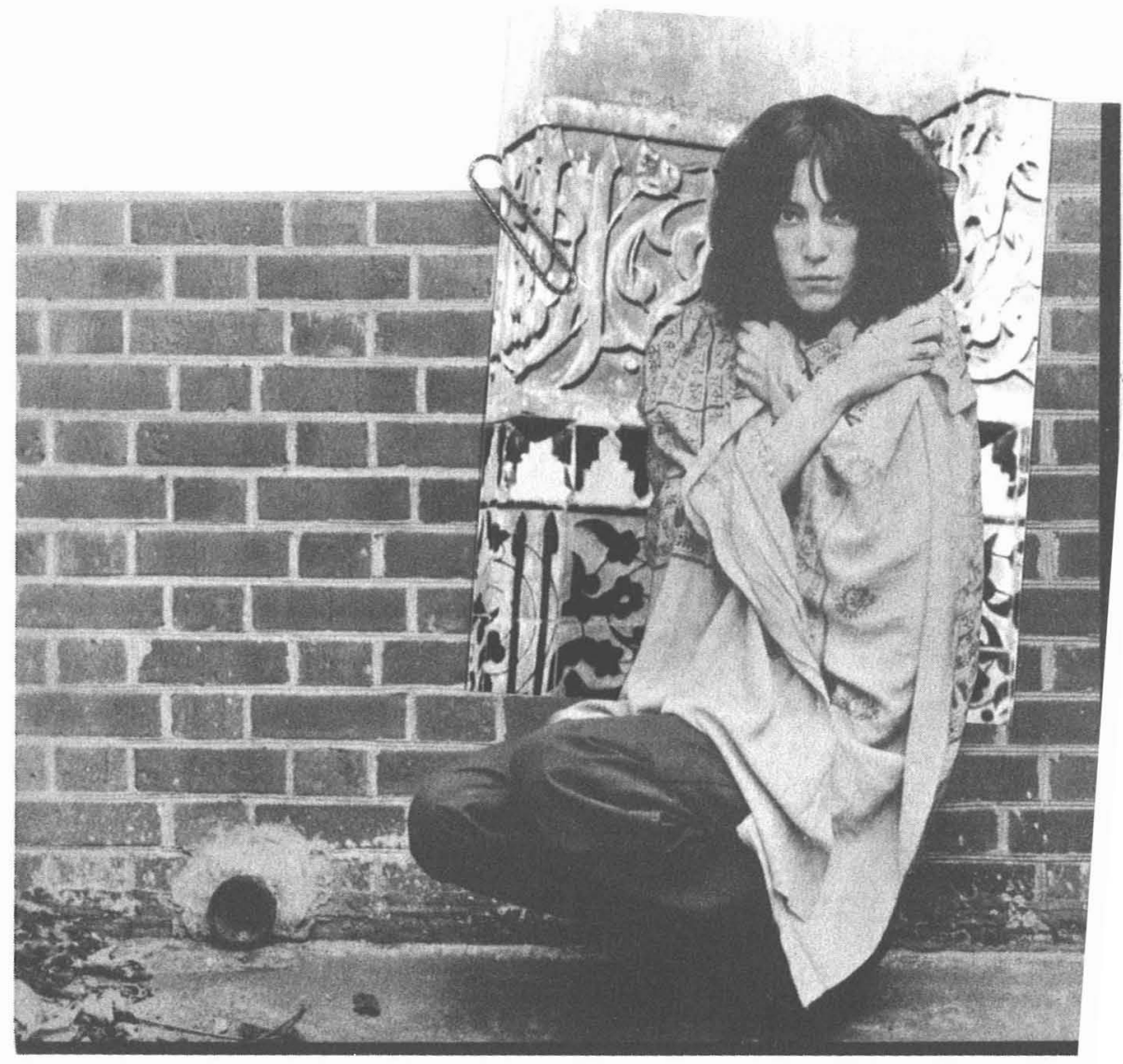

Illustration : Suzanne de Lotbinière-Harwood. 
Patti et moi

oh we had such a brainiac amour

dans la vitrine d'un magasin de disque à New York octobre 75. elle là debout/born in flames

stoned eyes search lights straight ahead

chemise blanche baudelaire tignasse \& cravate noires

les mains en beau geste sur la poitrine :

HORSES : "Jesus died for somebody's sins/but not mine"2

ni cartésienne ni barthésienne, je tremble devant des mots comme «critique», «théorie», «sémiotique». j’ai pourtant fait mon cours classique (ma transistor-sister dans la poche de mon uniforme)! en psychothérapie j'ai compris que ma totale incapacité à jouer aux échecs —panique, désorganisation intérieure, "hystérie»vient du rapport au Père: le logos, la cérébration linéaire-binaire, le left-brain. refus viscéral de me prêter à cette stratégie des rois. peur de la structure parce qu'elle a toujours voulu dire la leur. peur(e) patriarcale d'y perdre ma poésie - my "intuitive mania", my "percussive blood" (B). je ne sais pas ce que j'écris avant de l'écrire. j'aime ce danger, l'aventure irrationnelle. pourtant, mes auteures québécoises préférées sont pionnières sur le plan théorique. je veux être plus qu'une «bourgeoise qui fait des lettres". je devrai donc devenir (comme on «devient» femme) habile à exercer mon sens critique féministe en écriture comme ailleurs. "if we don't do it, somebody else will," me dit Gail Scott. 
PATTI LEE SMITH american rock poet, enfant terrible de décembre 46. première femme à prendre la parole

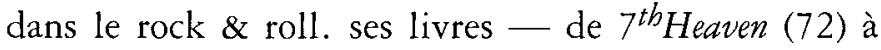
Babel (78) - ma première vraie lecture : enfin une ouverture! "the first book that filled me with ecstasy" (B). confirmation de la possibilité de mon projet hallucinatoire : devenir femme poète rock. ses disques Horses, Radio Etbiopia, Easter, Wave - sa poésie ambiante, de quoi me mettre sous la dent sur le stéréo. she BOP kept rigor mortis from setting in. ravissement de la la la découverte entrée dans une complicité passionnée avec $\mathrm{J}$. qui lui ressemble, qui m'achète ses livres à NYC. «Dec. 10/77 - à Suzanne, pour ta collection - Love, J." shortly thereafter elle me laisse tomber. mon journal : "J. doesn't need me anymore ...". pour me venger, je lui vole Patti Smith, gros paperback de paroles et musique, photos et textes inédits. (depuis 79 , nous sommes sans nouvelles d'elle.)

***水必米米****

quand il s'agit du Québec, je ne sais si dire "we" ou "they"... en $81 \mathrm{j}$ 'ai dit "j'écris en français, je vis en anglais» ${ }^{3}$. changeons le $V$ pour un $L$ : j'écris en français, je lis en anglais. en $3^{e}$ année c'était le p'tit catéchisme et mes comix Katy Keene. pour moi la traduction c'est plus qu'une profession, c'est ma façon d'être. ma périlleuse identité de parfaite bilingue. "am $i$ all alone in this generation?" (Birdland, Horses). mon utopie : écrire comme je vis et parle et lis ... dans les-deux-langues à la fois/back \& forth. "after all, i am the last white nigger" (Rolling Stone, July 27/78). Cocteau dans la Difficulté d'être : "Plus un livre nous importe, plus mal 
nous le lisons. Notre substance s'y glisse et le pense à notre usage». ${ }^{4}$ so : what happens inside quand je traduis mes lectures dans mes textes? "the disintegration of brain into lingua into the pulse of rbythm" (Smith, HERoes, Hit Parader, april 78).

$* * * * * * * * * * * *$

vers 68 , ils m'ont traitée de «Traître !» à cause de mon nom, de mon anglais. mais le rock \& roll dans mes veines speaks white traduction-trahison? je n'ai jamais eu d'autre choix que de rejeter le nationalisme québécois qui voulait me faire choisir, donc me censurer. t'es pas capable d'écrire rien qu'en français ! c'est d'la facilité, tes citations en anglais ! personne va publier ça! "femalelfeel malelever since $i$ felt the need to chooseli'd cboose the maleli felt boy rhythms when $i$ was in knee punts/so $i$ stayed in pants ..." Seventh Heaven's.

dans l'ambisexualité de son personnage rock et de son oeuvre - sa célèbre version «aux femmes» de G-L-O-R-I-A, ses poèmes d'amours lesbiens et hétéro dans son mysticisme nigredolalbedolrubedo ${ }^{6}$, je voyais reflétée ma propre ambiguité linguistique/culturelle/ spirituelle. toute écartillée, j'enviais sa réussite à s'intégrer son sens érotique de la réalité. "ber treasure was the absolute beam of concentration" $(B)$.

mon écriture que je reportais sans cesse (censure) dans mon journal était en sursis dans la sienne. je ré-écrivais mes livres au lieu d'écrire le mien. j'ai fait plus qu'y glisser ma substance. ma faim d'elle a fini par faire le vide sur la page. elle est devenue ma substance.

j'enviais son nom. "easy to spell, hard to forget" disait J. i mean a-t-on idée de naître avec un nom bilingue à 
trait d'union - qui unit/sépare quoi ? de Lotbinière parlerait féminin et Harwood masculin?

l'oblique adoptée d'elle serait la marque grammaticale de ma séparation.

"my body encases a soul split and contradictory. good and evil, disciplined and maniac. i offer no excuses or explanations. $i$ am a physical architect building a temple of experience. $i$ am still gathering information". (Melody Maker, March 18/78).

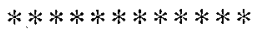

terrorisée par la tâche d'écrire ce texte critique, j'angoisse je me défonce je rêve un film intitulé «PLATINUM HELL», équation psychique entre Patti et $J$. je glisse en pleine émotion lancinante de cette rupture ayant eu son oeuvre poétique et musicale comme fondde-scène. les structures mentales que j'essaie de formuler se fondent en feelings, la théorie serait une affectivité ? paralysée, j'arrête d'écrire pendant trois semaines. ai-je à mon insu attrapé la vieille résistance anglosaxonne face à la théorie ? pénible mais l'orage passe... je la lis je l'écoute je re-connecte avec ce-pourquoi j'écris : pour parler de $M y-M y-M y$ Generation Québecrock - quelle est ma/notre culture ? quelle est ma/ notre moralité, en la dérive fin-de-siècle de notre maturité ? et pour dé-dire la répression de la divinité dans son être femme.

hy-brides "bigh on rebellion" (Easter), elle m'a permis de continuer d'espérer, elle qui aussi avait longtemps été just a fan du rock et de l'écriture, d'aspirer à incarner mon rêve-vêtement de peau. le féminisme m’a donné 
les structures intérieures nécessaires pour passer à l'acte, "true to a position of vision". (B).

changeons le $\mathrm{r}$ pour un 1 . cérébration devient célébration

\section{NOTES}

${ }^{1}$ Les citations marquées (B) dans le texte sont tirées de Patti Smith, Babel, Putnam, NY, 1978.

2 Les deux phrases en italique dans ce paragraphe sont tirées du long-jeu Horses (Arista 4066, 1975) de Patti Smith.

3 S. de L.-Harwood, «État d'esprit New York» in NBJ-la Femme et la Ville. numéro 102, avril 1981.

4 Jean Cocteau, la Difficulté d'être, Collection 10/18, 1957. «De la lecture», pp. 61-65.

5 Patti Smith, Seventh Heaven, Telegraph Books, Boston, 1972.

${ }^{6}$ noir/blanc/rouge : les trois stades initiatiques en alchimie. 